\title{
Any Monotone Property of 3-uniform Hypergraphs is Weakly Evasive
}

\author{
Raghav Kulkarni ${ }^{1}$, Youming Qiao ${ }^{31}$, and Xiaoming Sun ${ }^{2 \star}$ \\ 1 Centre for Quantum Technologies, the National University of Singapore ${ }^{\star \star}$ \\ \{kulraghav, jimmyqiao86\}@gmail.com \\ 2 Institute of Computing Technology, Chinese Academy of Sciences \\ sunxiaoming@ict.ac.cn \\ ${ }^{3}$ Centre for Quantum Computation and Intelligent Systems, University of \\ Technology, Sydney
}

\begin{abstract}
For a Boolean function $f$, let $D(f)$ denote its deterministic decision tree complexity, i.e., minimum number of (adaptive) queries required in worst case in order to determine $f$. In a classic paper, Rivest and Vuillemin [19] show that any non-constant monotone property $\mathcal{P}$ : $\{0,1\}\left(\begin{array}{l}n \\ 2\end{array}\right) \rightarrow\{0,1\}$ of $n$-vertex graphs has $D(\mathcal{P})=\Omega\left(n^{2}\right)$.

We extend their result to 3 -uniform hypergraphs. In particular, we show that any non-constant monotone property $\mathcal{P}:\{0,1\}\left(\begin{array}{l}n \\ 3\end{array}\right) \rightarrow\{0,1\}$ of $n$ vertex 3-uniform hypergraphs has $D(\mathcal{P})=\Omega\left(n^{3}\right)$.

Our proof combines the combinatorial approach of Rivest and Vuillemin with the topological approach of Kahn, Saks, and Sturtevant. Interestingly, our proof makes use of Vinogradov's Theorem (weak Goldbach Conjecture), inspired by its recent use by Babai et. al. [1] in the context of the topological approach. Our work leaves the generalization to $k$-uniform hypergraphs as an intriguing open question.
\end{abstract}

Keywords: decision tree complexity; hypergraph properties; evasiveness; query complexity; group actions

\section{Introduction}

The decision tree model aka query model [3], perhaps due to its simplicity and fundamental nature, has been extensively studied over decades; yet there remain some outstanding open questions about it.

Fix a Boolean function $f:\{0,1\}^{n} \rightarrow\{0,1\}$. A deterministic decision tree $D_{f}$ for $f$ takes $x=\left(x_{1}, \ldots, x_{n}\right)$ as an input and determines the value of $f\left(x_{1}, \ldots, x_{n}\right)$

* Part of this work was done while the author was visiting the Centre for Quantum Techologies, National University of Singapore. This work was supported in part by the National Natural Science Foundation of China Grant 61170062, 61222202.

** Research at the Centre for Quantum Technologies is funded by the Singapore Ministry of Education and the National Research Foundation. 
using queries of the form "is $x_{i}=1$ ?". Let $C\left(D_{f}, x\right)$ denote the cost of the computation, that is the number of queries made by $D_{f}$ on input $x$. The deterministic decision tree complexity of $f$ is defined as $D(f)=\min _{D_{f}} \max _{x} C\left(D_{f}, x\right)$.

The function $f$ is called evasive if $D(f)=n$, i.e., one must query all the variables in worst case in order to determine the value of the function.

\subsection{The Anderaa-Rosenberg-Karp Conjecture}

A Boolean fuction $f$ is said to be monotone (increasing) if for any $x \leq y$ we have $f(x) \leq f(y)$, where $x \leq y$ iff for all $i: x_{i} \leq y_{i}$. A property of $n$-vertex graphs is a Boolean function $\mathcal{P}:\{0,1\}^{\left(\begin{array}{l}n \\ 2\end{array}\right)} \rightarrow\{0,1\}$ whose variables are identified with the $\left(\begin{array}{l}n \\ 2\end{array}\right)$ potential edges of $n$-vertex graphs and the function $\mathcal{P}$ is invariant under relabeling of the vertices. $\mathcal{P}(G)=0$ means that the graph $G$ satisfies the property. A natural theme in the study of decision tree complexity is to exploit the structure within $f$ to prove strong lower bounds on its query complexity. A classic example is the following conjecture attributed to Anderaa, Rosenberg, and Karp, asserting the evasiveness of monotone graph properties:

Conjecture 1 (ARK Conjecture). (cf. [8]) Every non-trivial monotone graph property is evasive.

Some natural examples of monotone graph properties are: connectedness, planarity, 3-colorability, containment of a fixed subgraph etc.

Since its origin around 1975, the ARK Conjecture has caught the imagination of generations of researchers resulting in beautiful mathematical ideas; yet - to this date - remains unsolved. A major breakthrough on ARK Conjecture was obtained by Kahn, Saks, and Sturvevant [8] via their novel topological approach. They settled the conjecture when the number of vertices of the graphs is a power of prime number. The topological approach subsequently turned out useful for solving some other variants and special cases of the conjecture. For example: Yao confirms the variant of the conjecture for monotone properties of bipartite graphs [25]. More recently, building on Chakraborty, Khot, and Shi's work [4], Babai et. al. [1] show that under some well-known conjectures in number theory, forbidden subgraph property - containment of a fixed subgraph in the graph - is evasive. We refer the readers to the lecture notes [12] by Lovász and Young for a nice exposition of the works around this topic.

\subsection{The Evasiveness Conjecture}

The key feature of monotone graph properties is that they are sufficiently symmetric. In particular, they are transitive Boolean functions, i.e., there is a group acting transitively on the set of variables under which the function remains invariant. A natural question was raised: how much symmetry is necessary in order to guarantee the evasiveness? The following generalization (cf. [13]) of ARK Conjecture asserts that only transitivity suffices. 
Conjecture 2 (Evasiveness Conjecture $(E C)$ ). If $f$ is a non-trivial monotone transitive Boolean function, then $f$ is evasive.

Rivest and Vuillemin [19] confirm the above conjecture when the number of variables is a power of prime number. The general case remains widely open.

\subsection{The Weak Evasiveness Conjecture}

Recently Kulkarni [7] proposes to investigate the following:

Conjecture 3 (Weak Evasiveness Conjecture). If $\left\{f_{n}\right\}$ is a sequence of non-trivial monotone transitive Boolean functions then for every $\epsilon>0$

$$
D\left(f_{n}\right) \geq n^{1-\epsilon} .
$$

The best known lower bound in this context is $D(f) \geq R(f) \geq n^{2 / 3}$, which follows from the work of O'Donnell et. al. [18]. It turns out that [7] the above conjecture is equivalent to the EC! Furthermore: the Rivest and Vuillemin [19] result, which settles the ARK conjecture up to a constant factor, in fact confirms the Weak-EC for graph properties.

Theorem 1 (Rivest and Vuillemin). If $\mathcal{P}:\{0,1\}\left(\begin{array}{l}n \\ 2\end{array}\right) \rightarrow\{0,1\}$ is a non-trivial monotone property of graphs on $n$ vertices then $D(\mathcal{P})=\Omega\left(n^{2}\right)$.

It is interesting to note that the proof of equivalence in Kulkarni [7] does not hold between ARK and Weak-ARK. Hence: even though Weak-ARK is settled, the ARK is still wide open.

\subsection{Our results on the Weak EC}

In this paper we prove an analogue of Rivest and Vuillemin's result (Theorem 1) for 3-uniform hypergraphs. A property of 3-uniform hypergraphs on $n$ vertices is a Boolean function $\mathcal{P}:\{0,1\}\left(\begin{array}{l}n \\ 3\end{array}\right) \rightarrow\{0,1\}$ whose variables are labeled by the $\left(\begin{array}{l}n \\ 3\end{array}\right)$ potential edges of $n$-vertex 3 -uniform hypergraphs and $\mathcal{P}$ is invariant under relabeling of the vertices.

Theorem 2. If $\mathcal{P}:\{0,1\}^{\left(\begin{array}{l}n \\ 3\end{array}\right)} \rightarrow\{0,1\}$ is a non-trivial monotone property of 3-uniform hypergraphs on $n$ vertices, then

$$
D(\mathcal{P})=\Omega\left(n^{3}\right)
$$

Our proof technique can be briefly described as follows: First we combine the combinatorial approach of Rivest and Vuillemin with the topological approach of Kahn, Saks, and Sturtevant to prove the result when $n=3^{k}$. Then we use the $3^{k}$ case to prove the result for arbitrary $n$ via an interesting application of 
the famous Vinogradov's Theorem that asserts that every odd integer can be expressed as sum of three prime numbers.

Interestingly, we do not yet know how to generalize our proof technique to $k$-uniform hypergraphs. But in this context we are able to prove a partial result on 4-uniform hypergraphs.

Theorem 3. Let $\mathcal{P}:\{0,1\}^{n \times n \times n \times n} \rightarrow\{0,1\}$ be a 4-uniform 4-partite hypergraph property of 4 -vertex hypergraphs. If $\mathcal{P}$ is non-trivial and monotone, then

$$
D(\mathcal{P})=\Omega\left(n^{4}\right)
$$

The organisation of this paper is as follows. Section 2 contains the preliminaries. Section 3 contains the proof of $n=3^{k}$ case. Section 4 uses $3^{k}$ case to prove the general case, in particular it contains the proof of Theorem 2. Section 5 contains some partial results for 4 -uniform hypergraphs. Section 6 contains conclusion and open ends.

\section{Preliminaries}

In this paper $[n]$ denotes the set $\{1, \ldots, n\}$.

\subsection{Rivest-Vuillemin: combinatorial approach}

In a beautiful paper, Rivest and Vuillemin show that the ARK Conjecture holds up to a constant factor, i.e., any non-trivial monotone graph property is weakly evasive. As an intermediate step [19] show the following:

Theorem 4 (Rivest-Vuillemin). If $n$ is a power of a prime number and $f$ : $\{0,1\}^{n} \rightarrow\{0,1\}$ is any function invariant under a transitive permutation group such that $f(0, \ldots, 0) \neq f(1, \ldots, 1)$, then $D(f)=n$.

In this paper we prove the weak-evasiveness of monotone properties of 3uniform hyper-graphs, which extends the result of Rivest and Vuillemin for graph properties. Our proof is inspired by the one by Rivest and Vuillemin. Interestingly we use, in addition to the combinatorial approach of Rivest and Vuillemin, the powerful topological approach of Kahn, Saks, and Sturtevant combined with a deep theorem in number theory.

\subsection{Kahn-Saks-Sturtevant: topological approach}

In a seminal paper, Kahn, Saks, and Sturtevant [8] introduce a novel topological approach to settle the ARK Conjecture when the number of vertices of graphs is a power of a prime number. Their crucial observation was that non-evasiveness of monotone properties has a strong topological consequence, namely the corresponding simplicial complex is contractible to a point. Further they exploit this topological consequence via Oliver's Fixed Point Theorem [17] under the actions of certain special type of groups. 
We say that a group $\Gamma$ satisfies Olivers Condition if there exist (not necessarily distinct) primes $p, q$ such that $\Gamma$ has a (not necessarily proper) chain of subgroups $\Gamma_{2} \triangleleft \Gamma_{1} \triangleleft \Gamma$ such that $\Gamma_{2}$ is a $p$-group, $\Gamma_{1} / \Gamma_{2}$ is cyclic, and $\Gamma / \Gamma_{1}$ is a $q$-group, where $p$-group means a group whose order is a power of a prime $p$.

Theorem 5 (Kahn-Saks-Sturtevant). If $\Gamma$ satisfies Oliver's Condition and acts transitively on the set $S$ of variables, then for any non-trivial monotone $\Gamma$-invariant function $f:\{0,1\}^{S} \rightarrow\{0,1\}$, we have: $D(f)=|S|$.

Kahn, Saks, and Sturtevant made the assumption that the number of vertices of the graph - $n$ is a prime power and used the following group that satisfies Oliver's Condition:

$$
A F F(n):=A G L(1, n),
$$

the group of affine transformations $x \mapsto a x+b$ over the field $\mathbb{F}_{n}$ of order $n$; $a \in \mathbb{F}_{n}-\{0\}, b \in \mathbb{F}_{n}$. The two key properties of this group are that it is a cyclic extension of a $p$-group, i.e., it satisfies Oliver's Condition; moreover it acts doubly transitively on $[n]$, i.e., any $(i, j)$ can be mapped to any $\left(i^{\prime}, j^{\prime}\right)$ for $i \neq j$ and $i^{\prime} \neq j^{\prime}$.

In this paper, we make use of the $A F F(n)$, as well as another group-theoretic construction called wreath product. We recall the definition, and refer the readers to [20, Section 1.6] detailed discussion. For a finite set $S$, let $\operatorname{Sym}(S)$ be the symmetric group on $S$. Let $G \leq \operatorname{Sym}(S)$ and $H \leq \operatorname{Sym}(T)$. The wreath product $G$ ? $H$ is a permutation group acting on $S \times T$, defined as follows. The base group of the wreath product is the direct product $G^{T}$, that is $|T|$ copies of $G$. For $t \in T$, the $G_{t}$ independently acts on the corresponding copy $S \times\{t\}$. Specifically, for $(\omega, \delta) \in S \times T$, and $f \in G^{T},(s, t)^{f}=\left(s^{f(t)}, t\right)$. $G \nmid H$ also contains a subgroup $H^{*}$ isomorphic to $H$, acting only on the second component of $S \times T$. That is for $h \in H,(s, t)^{h}=\left(s, t^{h}\right)$. G $\succ H$ is the group generated by $G^{S}$ and $H^{*}$.

\subsection{Prime-partition via Vinogradov's Theorem}

The Goldbach Conjecture asserts that every even integer can be written as the sum of two primes. Vinogradov's Theorem [24] says that every sufficiently large odd integer $m$ is the sum of three primes $m=p_{1}+p_{2}+p_{3}$. We use here Haselgroves version [5] of Vinogradov's theorem which states that we can require the primes to be roughly equal: $p_{i} \sim m / 3$. This can be combined with the Prime Number Theorem to conclude that every sufficiently large even integer $m$ is a sum of four roughly equal primes.

This fact was first used by Babai et. al. [1] to construct the group actions satisfying Oliver's Condition in order to show that any monotone property of sparse graphs is evasive.

\section{3-uniform Hypergraphs: $n=3^{k}$}

We prove the following theorem in this section. 
Theorem 6. Let $n=3^{k}$, and $\mathcal{P}$ be 3-uniform hypergraph property of $n$-vertex hypergraphs. If $\mathcal{P}$ is non-trivial and monotone, then $D(\mathcal{P})=\Omega\left(n^{3}\right)$.

Proof. Our proof strategy is inspired by the one by Rivest and Vuillemin's proof that non-trivial and monotone graph properties of graphs with $n=2^{k}$ vertices are weakly evasive. The basic strategy is to set up a family of graphs $G_{0} \subset$ $G_{1} \subset \ldots \subset G_{k}$, among which there are two adjacent graphs $G_{\ell}$ and $G_{\ell+1}$ such that $G_{\ell}$ satisfies $\mathcal{P}$ whereas $G_{\ell+1}$ does not. Now we start with the smaller graph $G_{\ell}$ and gradually add edges to it under the assumption that $D(\mathcal{P})$ is not $\Omega\left(n^{3}\right)$ and conclude that even after adding these edges the property $\mathcal{P}$ is satisfied. Eventually, after adding sufficiently many edges this would lead to a contradiction as we would be able to conclude that $G_{\ell+1}$ satisfies the property.

Rivest and Vuillemin choose $G_{\ell}$ to be the disjoint union of $2^{n-\ell}$ cliques on $2^{\ell}$ vertices. Further they use Theorem 4 to add the edges to finally lead to a contradiction. Similar to Rivest and Vuillemin, we start our proof by using Theorem 4 to add certain type of edges. However, while handling the 3-uniform hypergraph properties, we face more complications. The natural choice of $G_{i}$ to be disjoint union of hyper-cliques seems to fail and Theorem 4 seems inadequate in dealing with all types of edges. We overcome this obstacle by suitably changing the family of graphs and by making use of the topological approach of Kahn, Saks, and Sturtevant (Theorem 5) to deal with the other type of edges.

\subsection{Our choice of the graph family: cliques with spikes}

To prove the theorem we consider the following family of hypergraphs on $n$ vertices. For $j \in\{0,1, \ldots, k\}$, let $G_{j}$ be the hypergraph defined as follows: firstly $G_{j}$ contains a disjoint union of $3^{k-j}$ copies of cliques on $3^{j}$ vertices. Then if an edge $\{u, v, w\}$ satisfies that $u, v$ are in the one clique while $w$ is in another one, it is also included in $G_{j}$. We call such edges spikes.

As $G_{0}$ is the empty hypergraph, and $G_{k}$ is the complete hypergraph, we see that $G_{0}$ satisfies $\mathcal{P}$ while $G_{k}$ does not as $\mathcal{P}$ is non-trivial. This suggests that there exists $\ell \in\{0,1, \ldots, k-1\}$ such that $G_{\ell}$ satisfies the property while $G_{\ell+1}$ does not as $\mathcal{P}$ is monotone.

Now collect the cliques in $G_{\ell}$ into three groups $V_{1} \cup V_{2} \cup V_{3}$, each group containing $3^{k-\ell-1}$ cliques. We then consider the property $\mathcal{P}_{1}$ induced by $\mathcal{P}$ after fixing the values at the edges $\left\{\{u, v, w\} \mid u, v, w \in V_{i}\right.$, or $\left.\{u, v, w\} \in G_{\ell}\right\}$ as in $G_{\ell}$. Note that $\mathcal{P}_{1}$ is a non-trivial property, because $\mathcal{P}$ is monotone and the graph $G_{\ell+1}$ is contained in the graph $G_{\ell} \bigcup E$ where $E$ denotes the edges corresponding to the domain of $\mathcal{P}_{1}$.

\subsection{Two types of edges}

The edges not fixed in $\mathcal{P}_{1}$ are of two types:

Type $1 T_{1}=\left\{\left\{v_{1}, v_{2}, v_{3}\right\} \mid v_{i} \in V_{i}, i \in[3]\right\}$.

Type $2 T_{2}=\left\{\{u, v, w\} \mid u, v \in V_{i}, w \in V_{j}, i \neq j\right\}$. Note that $v, w$ cannot come from the same clique otherwise it would have been fixed. 


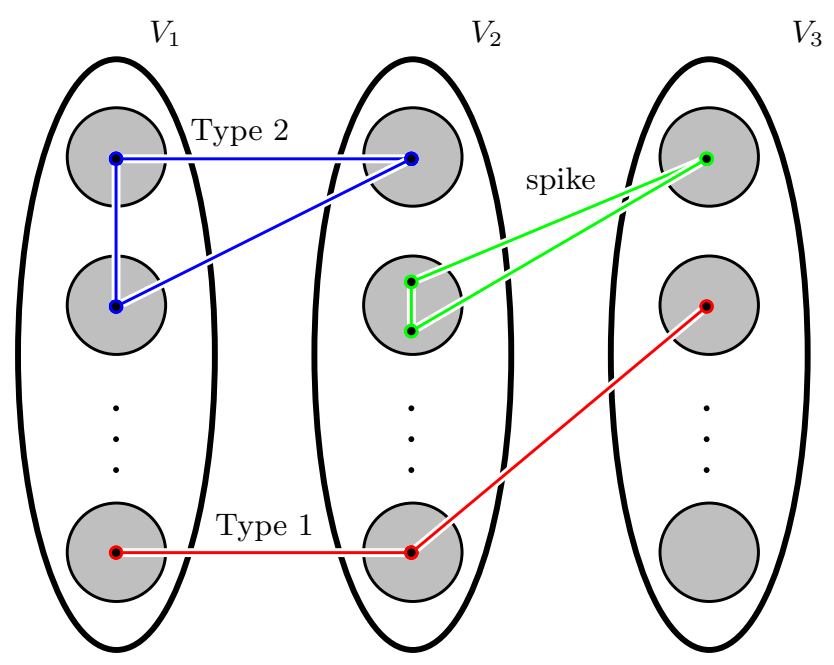

Fig. 1. An illustration of the spike (green), and Type 1 (red) and Type 2 (blue) edges.

Before going on we define two group actions on $V_{1}$. Firstly, $H_{1}=\mathbb{Z}_{3^{\ell}}<\mathbb{Z}_{3^{k-1-\ell}}$ acts on $V_{1}$, where $3^{k-1-\ell}$ copies of $\mathbb{Z}_{3}$ act independently on the $3^{k-1-\ell}$ cliques, and $\mathbb{Z}_{3^{k-1-\ell}}$ permutes among the cliques. Secondly, we define the group action of $H_{2}=\mathbb{Z}_{3^{\ell}}$ ? $A F F\left(3^{k-1-\ell}\right)$ on $V_{1}$ similarly to $H_{1}$. That is, $3^{k-1-\ell}$ copies of $\mathbb{Z}_{3^{\ell}}$ act independently on the $3^{k-1-\ell}$ cliques, and $A F F\left(3^{k-1-\ell}\right)$ acts on the cliques in doubly-transitive way. Recall that for a vector space $V, A F F(V)$ is the affine group on $V . H_{1}$ and $H_{2}$ are both subgroups of the automorphism group of induced subgraph of $G_{\ell}$ on $V_{1}$. Then note that $H_{1}$ is a 3 -group, and $H_{2}$ is transitive on $\{\{u, v\} \mid u$ and $v$ are from different cliques $\}$. Finally, it can be verified that $H_{2}$ belongs to the group class as in Theorem 5 .

Table 1. Groups used to handle Type 1 and Type 2 edges

Type $1\left(\mathbb{Z}_{3 \ell} \nmid \mathbb{Z}_{3^{k-1-\ell}}\right) \times\left(\mathbb{Z}_{3^{\ell}} \nmid \mathbb{Z}_{3^{k-1-\ell}}\right) \times\left(\mathbb{Z}_{3^{\ell}} \nmid \mathbb{Z}_{3^{k-1-\ell}}\right)$ Type $2\left(\mathbb{Z}_{3^{\ell}} \succ A F F\left(3^{k-1-\ell}\right)\right) \times\left(\mathbb{Z}_{3^{\ell}} \imath \mathbb{Z}_{3^{k-1-\ell}}\right)$

\subsection{Adding Type 1 edges}

Now we consider the property $\mathcal{P}_{2}$ induced by $\mathcal{P}_{1}$ by setting Type 2 edges to be absent. Note that the number of Type 1 edges is $3^{3(k-1)}$, thus a prime power. Let $H_{1} \times H_{1} \times H_{1}$ act on $V_{1} \times V_{2} \times V_{3}$ in a natural way: each copy of $H_{1}$ acts on vertices of $V_{i}$ independently. It is seen that this action preserves the fixed subgraph, and $\mathcal{P}_{2}$ is invariant under this action. If after adding all Type 1 edges $\mathcal{P}_{2}$ would $n o t$ be satisfied, then by the Rivest-Vuillemin theorem, $\mathcal{P}_{2}$ is evasive. That is $D(\mathcal{P}) \geq D\left(\mathcal{P}_{2}\right)=3^{3(k-1)}=\Omega\left(n^{3}\right)$ and we would be done. 


\subsection{Adding Type 2 edges}

Let $\mathcal{P}_{3}$ be the property induced by $\mathcal{P}_{1}$ by setting Type 1 edges to be present. The discussion from last paragraph suggests that $\mathcal{P}_{3}$ is a non-trivial property, and note that $\mathcal{P}_{3}$ only has Type 2 edges left unfixed. For $i, j \in[3], i \neq j$, let $T_{2}(i, j)=\left\{\{u, v, w\} \mid u, v \in V_{i}, w \in V_{j}\right\}$. Let $\mathcal{P}_{4}$ be the property induced by $\mathcal{P}_{3}$ by setting edges in $T_{2} \backslash T_{2}(1,2)$ to be absent. Note that $\left|T_{2}(1,2)\right|=\Omega\left(n^{3}\right)$.

(Oliver's Condition Holds) Consider the group $H=H_{2} \times H_{1}$ acting on $V_{1} \times V_{2}$ in a natural way. It is verified that $H$ preserves the structure of the fixed graph, and $\mathcal{P}_{3}$ is invariant under $H$. It is easy to check that $H$ belongs to the group class described in Theorem 5. This allows us to apply Theorem 5 (the Kahn, Saks and Sturtevant Theorem) to conclude that either $\mathcal{P}_{4}$ is trivial; if not then its query complexity is $\Omega\left(n^{3}\right)$ and we would be done.

(Orbits are large) Here we use a key property of the action of $H$, namely that the orbit of any edge is of large size: $\Omega\left(n^{3}\right)$. Thus we can add edges in $T_{2}(1,2)$ to get another restriction $\mathcal{P}_{5}$. Then we use the same group as above for $V_{2} \times V_{3}$ to add edges in $T_{2}(2,3)$.

\subsection{Deriving a contradiction}

Continuing this way we can keep adding $T_{2}(i, j)$ edges while maintaining that the hyper-graph still satisfies the property. But then we would get $G_{\ell+1}$ as a subgraph which by our choice of $\ell$, does not satisfy the property. Contradiction!

\section{3-uniform Hypergraphs: General $n$}

In this section we prove the main theorem.

Theorem 1.5, restated. If $\mathcal{P}:\{0,1\}\}^{\left(\begin{array}{l}n \\ 3\end{array}\right)} \rightarrow\{0,1\}$ is a non-trivial monotone property of 3-uniform hypergraphs on $n$ vertices, then $D(\mathcal{P})=\Omega\left(n^{3}\right)$.

Proof. The natural way of extending Rivest and Vuillemin's argument for 3uniform hyper graphs for arbitrary $n$ leads to analysis of several types of edges. We do not know an easy way to handle this via combinatorial approach. We can use the topological approach together with an interesting theorem about partitioning an integer into prime numbers to patch up the $3^{k}$ case to arbitrary $n$.

\subsection{Prime-partition of $\boldsymbol{n}$ via Vinogradov's Theorem}

We distinguish two cases: (Case 1) $n$ is even and (Case 2) $n$ is odd. Let us consider Case 1: $n$ is even. The other case can be handled in a similar fashion. Let $k$ be the largest power of 3 that does not exceed $n$. Since $n$ is even, we can write (using the above mentioned Hasegrove's version of Vinogradov's Theorem) $n=p_{1}+p_{2}+p_{3}+3^{k-1}$, where $p_{i}$ s are prime numbers and $p_{i} \sim p_{j}$. Moreoever: note that by our choice of $k$ we can assume: $p_{i} \leq 3^{k}$. 


\subsection{Patching up $3^{k}$ case to general $n$}

We partition $[n]$ into parts of size $p_{1}, p_{2}, p_{3}$ and $3^{k-1}$ as described in the previous section. Let $\mathcal{P}$ be a non-trivial monotone property of 3-uniform hyper-graphs on $n$ vertices. Theorem 6 allows us to conclude that either (a) $D(\mathcal{P})=\Omega\left(n^{3}\right)$ or (b) any $3^{k}$ vertex (hyper) clique satisfies $\mathcal{P}$. In Case (a) we are done. So let us assume that we are in Case (b). Since $p_{1} \leq 3^{k}$ and since $\mathcal{P}$ is monotone, we may assume that the clique on $p_{1}$ vertices satisfies the property. Now we assume that the clique on $p_{1}$ vertices is present and restrict our attention to the induced property $\mathcal{P}_{2}$ of 3 -uniform hypergraphs on $p_{2}+p_{3}+3^{k-1}$ vertices. Again using the fact that $p_{2} \leq 3^{k}$ we can assume that the clique on $p_{2}$ vertices is also present in addition to the clique on $p_{1}$ vertices. Now we move our attention to the induced property $\mathcal{P}_{2}$ on $p_{3}+3^{k-1}$ vertex graphs. In one more step, we can move our attention to the induced property $\mathcal{P}_{3}$ on $3^{k-1}$ graphs which assumes that the cliques on the $p_{1}, p_{2}$, and $p_{3}$ vertices are present. Finally, with the use of Theorem 6 , we can conclude that the clique on the $3^{k-1}$ vertices is also present; if not then we could already conclude $D(\mathcal{P})=\Omega\left(n^{3}\right)$.

\subsection{Two types of edges}

Now we have a restriction $\mathcal{P}^{\prime}$ of our original property $\mathcal{P}$ in which the cliques on $p_{1}, p_{2}, p_{3}$ and $3^{k-1}$ vertices are present. We partition the absent edges into two types:

Type A the three endpoints of the edges belong to different cliques;

Type $\mathbf{B}$ two of the three endpoints belong to one clique and the remaining endpoint belongs to a different clique.

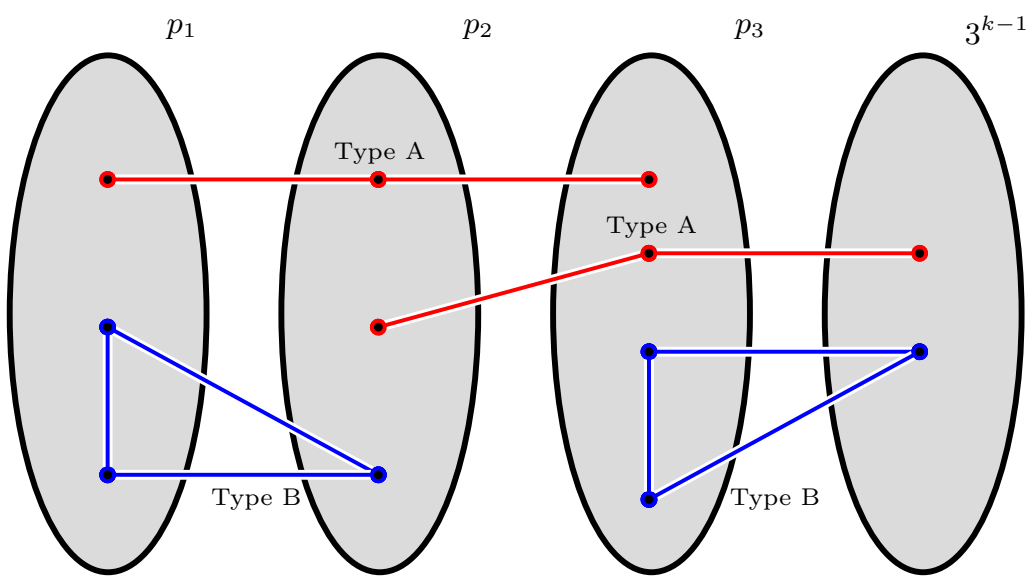

Fig. 2. An illustration of Type A (red) and Type B (blue) edges. 
Table 2. Groups used to handle Type A and Type B edges

\begin{tabular}{|l|l|}
\hline Type A & $\mathbb{Z}_{p_{1}} \times \mathbb{Z}_{p_{2}} \times \mathbb{Z}_{p_{3}}$, and $\mathbb{Z}_{p_{i}} \times \mathbb{Z}_{p_{j}} \times \mathbb{Z}_{3^{k-1}}$ \\
\hline Type B & $A F F\left(p_{i}\right) \times \mathbb{Z}_{p_{j}}$, and $A F F\left(p_{i}\right) \times \mathbb{Z}_{3^{k-1}}$, and $A F F\left(3^{k-1}\right) \times \mathbb{Z}_{p_{i}}$ \\
\hline
\end{tabular}

\subsection{Adding Type A edges}

Firstly: we conclude that all Type A edges must also be present; if not then $D(\mathcal{P})=\Omega\left(n^{3}\right)$. For this we use the following two types of groups: $\mathbb{Z}_{p_{1}} \times \mathbb{Z}_{p_{2}} \times \mathbb{Z}_{p_{3}}$ and $\mathbb{Z}_{p_{i}} \times \mathbb{Z}_{p_{j}} \times \mathbb{Z}_{3^{k-1}}$.

\subsection{Adding Type B edges}

Secondly: after adding all Type A edges we can conclude that all Type B edges must also be present; if not then $D(\mathcal{P})=\Omega\left(n^{3}\right)$. For this we use the following three types of groups: $A F F\left(p_{i}\right) \times \mathbb{Z}_{p_{j}}$ and $A F F\left(p_{i}\right) \times \mathbb{Z}_{3^{k-1}}$ and $A F F\left(3^{k-1}\right) \times \mathbb{Z}_{p_{i}}$.

(Oliver's Condition Holds) It is easy to check that all the groups that we use are the right ones for using the topological approach, i.e., they are " $q$-group extension of cyclic extension of $p$-groups," i.e., they satisfy Oliver's Condition.

(Orbits are large) A crucial property that we used in our proof is that the orbit of any edge under any of our group actions is large: $\Omega\left(n^{3}\right)$.

\subsection{Deriving a contradiction}

After adding both Type A and Type B edges to the cliques on the $p_{1}, p_{2}, p_{3}$ and $3^{k-1}$ vertices, we can conclude that the clique on $n$ vertices must satisfy $\mathcal{P}$; this contradicts with our initial assumption that $\mathcal{P}$ is non-trivial.

This completes the proof of Theorem 2 .

\section{4-uniform 4-partite Hypergraphs}

In this section we prove the weak evasiveness for properties of 4-uniform 4-partite hypergraphs. Theorem 1.6, restated. Let $\mathcal{P}:\{0,1\}^{n \times n \times n \times n} \rightarrow\{0,1\}$ be a 4 -uniform 4-partite hypergraph property of $4 n$-vertex hypergraphs. If $\mathcal{P}$ is nontrivial and monotone, then $D(\mathcal{P})=\Omega\left(n^{4}\right)$.

Proof: If $n$ is prime, the result directly follows from Theorem 4.

In the case when $n$ is not prime, let $p$ be a prime number such that $p<n<2 p$. Let $V=V_{1} \cup V_{2} \cup V_{3} \cup V_{4},\left|V_{i}\right|=n$ be the vertex set. The strategy is again by contrapositive: assume $D(\mathcal{P})$ is not of $\Omega\left(n^{4}\right)$. Then we shall start from the empty graph, and then add the edges with different types while keeping the value of the property not change. Finally we will get that the complete graph satisfies the property, which contradicts to the condition of being a non-trivial property.

Let $G_{0}$ be the empty graph; thus $f\left(G_{0}\right)=0$. Let $V_{i}=A_{i} \cup B_{i}$, where $A_{i}$ is a vertex set of size $p(i=1,2,3,4)$, and $B_{i}=V_{i} \backslash A_{i}$. 


\subsection{Adding edges in $A_{1} \times A_{2} \times A_{3} \times A_{4}$}

Consider a restriction $\mathcal{P}_{1}$ of $\mathcal{P}$ where all the variables outside $A_{1} \times A_{2} \times A_{3} \times A_{4}$ are set to be $0 . \mathcal{P}_{1}$ is a monotone transitive invariant function with $p^{4}$ variables, by Theorem $4 \mathcal{P}_{1}$ is trivial, otherwise $D\left(\mathcal{P}_{1}\right)=p^{4}=\Omega\left(n^{4}\right)$. Let $G_{1}$ be the graph with edges $A_{1} \times A_{2} \times A_{3} \times A_{4}$, thus $f\left(G_{1}\right)=0$.

\subsection{Adding edges in $B_{1} \times A_{2} \times A_{3} \times A_{4}$}

We will add all the edges in $B_{1} \times A_{2} \times A_{3} \times A_{4}$ to $G_{1}$, resulting in a graph $G_{2}$ with edges $V_{1} \times A_{2} \times A_{3} \times A_{4}$. Before doing that we consider a graph $G_{1}^{\prime}$ with edges $B_{1} \times A_{2} \times A_{3} \times A_{4}$. Since $p>n-p$, from the monotone and symmetry condition, we have $f\left(G_{1}^{\prime}\right) \leq f\left(G_{1}\right)=0$. Consider a restriction $\mathcal{P}_{2}$ of $\mathcal{P}$ where all the edges in $G_{1}^{\prime}$ are set to 1 and all the edges outside $V_{1} \times A_{2} \times A_{3} \times A_{4}$ are set to 0 . It is clear that $\mathcal{P}_{2}$ is a monotone transitive invariant function with $p^{4}$ variables, thus from Theorem $4 \mathcal{P}_{2}$ is a constant, otherwise $D\left(\mathcal{P}_{2}\right)=p^{4}=\Omega\left(n^{4}\right)$. Hence we get $f\left(G_{2}\right)=0$.

\subsection{Adding edges in $V_{1} \times B_{2} \times A_{3} \times A_{4}$}

Similar to the previous step, we first use the monotone and symmetry condition to "delete" some edges from $G_{2}$. Let $G_{2}^{\prime}$ be the graph with edges $V_{1} \times B_{2} \times A_{3} \times A_{4}$. From the monotone and symmetry condition, $f\left(G_{2}^{\prime}\right) \leq f\left(G_{2}\right)=0$. Consider the restriction $\mathcal{P}_{3}$ of $\mathcal{P}$ where all the edges in $G_{2}^{\prime}$ are set to 1 and all the edges outside $V_{1} \times V_{2} \times A_{3} \times A_{4}$ are set to 0 . It is easy to see that $\mathcal{P}_{3}$ can be further partitioned into two properties isomorphism to $\mathcal{P}_{1}$ and $\mathcal{P}_{2}$, respectively. By repeating the steps in Section 5.1 and 5.2 we conclude that $\mathcal{P}_{3}$ is trivial, otherwise $D\left(\mathcal{P}_{3}\right)=$ $\Omega\left(n^{4}\right)$. Hence $f\left(G_{3}\right)=0$, where $G_{3}$ is the graph with edges $V_{1} \times V_{2} \times A_{3} \times A_{4}$.

\section{Adding edges in $V_{1} \times V_{2} \times B_{3} \times A_{4}$ and $V_{1} \times V_{2} \times V_{3} \times B_{4}$}

These two steps are similar to the previous step, and we omit them here. After doing these steps, we get that the value of the complete graph is also 0 , which contradicts the non-trivial condition.

Remark 1. We note that the proof strategy for Theorem 1.6 can be extended to show that any $k$-uniform $k$-partite hypergraph property is weakly evasive, when $k$ is a constant. On the other hand, we do not know how to prove the weak evasiveness for 4-uniform hypergraph properties.

\section{Conclusion}

In this paper we are able to confirm a special case of the Weak-EC. In particular, we have shown that any non-trivial monotone property of 3-uniform hypergraphs is weakly evasive. It is interesting to see how far can one generalize our results.

Question 1. Is any non-trivial monotone property of $k$-uniform hypergraphs weakly evasive? 


\section{References}

1. László Babai, Anandam Banerjee, Raghav Kulkarni, Vipul Naik: Evasiveness and the Distribution of Prime Numbers. STACS 2010: 71-82

2. Itai Benjamini, Gil Kalai, and Oded Schramm: Noise sensitivity of Boolean functions and its application to percolation. Inst. Hautes tudes Sci. Publ. (MATH)., 90, 1999

3. Harry Buhrman, Ronald de Wolf: Complexity measures and decision tree complexity: a survey. Theor. Comput. Sci. 288(1): 21-43 (2002)

4. Amit Chakrabarti, Subhash Khot, Yaoyun Shi: Evasiveness of Subgraph Containment and Related Properties. SIAM J. Comput. 31(3): 866-875 (2001)

5. Haselgrove, C. B.: Some theorems on the analytic theory of numbers. J. London Math. Soc. 36 (1951) 273-277

6. Thomas P. Hayes, Samuel Kutin, Dieter van Melkebeek: The Quantum Black-Box Complexity of Majority. Algorithmica 34(4): 480-501 (2002)

7. Raghav Kulkarni: Evasiveness Through A Circuit Lens. To appear in ITCS 13 (2013)

8. Jeff Kahn, Michael E. Saks, Dean Sturtevant: A topological approach to evasiveness. Combinatorica 4(4): 297-306 (1984)

9. Eyal Kushilevitz, Yishay Mansour: Learning Decision Trees Using the Fourier Spectrum. SIAM J. Comput. 22(6): 1331-1348 (1993)

10. Raghav Kulkarni, Miklos Santha: Query complexity of matroids. Electronic Colloquium on Computational Complexity (ECCC) 19: 63 (2012)

11. Nathan Linial, Yishay Mansour, Noam Nisan: Constant Depth Circuits, Fourier Transform, and Learnability. J. ACM 40(3): 607-620 (1993)

12. László Lovász, Neal E. Young: Lecture Notes on Evasiveness of Graph Properties. CoRR cs.CC/0205031: (2002)

13. Frank H. Lutz: Some Results Related to the Evasiveness Conjecture. Comb. Theory, Ser. B 81(1): 110-124 (2001)

14. Ashley Montanaro, Tobias Osborne: On the communication complexity of XOR functions CoRR abs/0909.3392: (2009)

15. Noam Nisan, Mario Szegedy: On the Degree of Boolean Functions as Real Polynomials. Computational Complexity 4: 301-313 (1994)

16. Noam Nisan, Avi Wigderson: On Rank vs. Communication Complexity. Combinatorica 15(4): 557-565 (1995)

17. Oliver, R.: Fixed-point sets of group actions on finite acyclic complexes. Comment. Math. Helv. 50 (1975), 155-177

18. Ryan O'Donnell, Michael E. Saks, Oded Schramm, Rocco A. Servedio: Every decision tree has an influential variable. FOCS 2005: 31-39

19. Ronald L. Rivest, Jean Vuillemin: On Recognizing Graph Properties from Adjacency Matrices. Theor. Comput. Sci. 3(3): 371-384 (1976)

20. Derek J.S. Robinson. A Course in the Theory of Groups. Springer, 2nd edition, 1996.

21. Michael E. Saks, Avi Wigderson: Probabilistic Boolean Decision Trees and the Complexity of Evaluating Game Trees FOCS 1986: 29-38

22. Yaoyun Shi, Zhiqiang Zhang: Communication Complexities of XOR functions CoRR abs/0808.1762: (2008)

23. Zhiqiang Zhang, Yaoyun Shi: On the parity complexity measures of Boolean functions. Theor. Comput. Sci. 411(26-28): 2612-2618 (2010) 
24. Vinogradov, I. M.: The Method of Trigonometrical Sums in the Theory of Numbers (Russian). Trav. Inst. Math. Steklo 10, (1937)

25. Andrew Chi-Chih Yao: Monotone Bipartite Graph Properties are Evasive. SIAM J. Comput. 17(3): 517-520 (1988) 